DOI: $10.15393 / \mathrm{j} 3 . \operatorname{art} .2021 .10110$

UDC 517.521

\author{
Ç. KAMBAK, İ. ÇANAK
}

\title{
NECESSARY AND SUFFICIENT TAUBERIAN CONDITIONS UNDER WHICH CONVERGENCE FOLLOWS FROM SUMMABILITY $A^{r, p}$
}

\begin{abstract}
In this paper, we introduce the summability method $A^{r, p}$ and obtain necessary and sufficient Tauberian conditions under which the ordinary convergence of a sequence follows from its summability $A^{r, p}$. The main results are new Tauberian theorems for the summability method $A^{r, p}$, which are generalizations of the corresponding Tauberian theorems for the summability method $A^{r}$ introduced by Başar.
\end{abstract}

Key words: summability by $A^{r, p}$ method, slow oscillation, slow decrease, Tauberian condition

2010 Mathematical Subject Classification: 40E05, 40G05

1. Introduction. Let $p=\left(p_{n}\right)$ be a sequence of non-negative numbers with $p_{0}>0$ and

$$
P_{n}=\sum_{k=0}^{n} p_{k} \rightarrow \infty, \quad n \rightarrow \infty .
$$

Let $0<r<1$. The class $A^{r, p}=\left(a_{n k}^{r, p}\right)$ of Toeplitz matrices is given by

$$
a_{n k}^{r, p}=\left\{\begin{array}{lll}
\frac{p_{k}\left(1+r^{k}\right)}{P_{n}} & \text { if } \quad 0 \leqslant k \leqslant n \\
0 & \text { if } \quad k>n .
\end{array}\right.
$$

Given a sequence $x=\left(x_{n}\right)$ of real or complex numbers, we define the $A^{r, p}$ transform of $x$ by

$$
\left(A^{r, p} x\right)_{n}=\sigma_{n, p}^{r}(x)=\frac{1}{P_{n}} \sum_{k=0}^{n} p_{k}\left(1+r^{k}\right) x_{k}, n=0,1,2, \ldots
$$

(c) Petrozavodsk State University, 2021 
If

$$
\lim _{n \rightarrow \infty} \sigma_{n, p}^{r}(x)=l,
$$

we say that $\left(x_{n}\right)$ is summable to $l$ by summability $A^{r, p}$.

It is clear that (1) is a necessary and sufficient condition that every

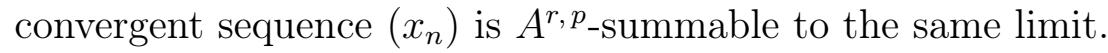

It is easy to check that if the limit

$$
\lim _{n \rightarrow \infty} x_{n}=l
$$

exists, we also have (2). However, the opposite is not true in general. Let us define the sequence $\left(x_{n}\right)$ by $x_{n}=(-1)^{n}\left(\left(1+r^{n}\right) p_{n}\right)^{-1}$ and particularly choose $p_{n}=(n+1)^{-1}$ for all non-negative integers $n$; then we have $\sigma_{n, p}^{r} \rightarrow 0$ as $n \rightarrow \infty$. This shows that $\left(x_{n}\right)$ is $A^{r, p}$-summable to zero, though it does not converge. Note that (2) implies (3) under the certain condition on the sequence $\left(x_{n}\right)$, called Tauberian condition. Any theorem that states that convergence of sequences follows from its $A^{r, p}$-summability and some Tauberian condition(s) is said to be a Tauberian theorem for summability method $A^{r, p}$.

If $p_{n}=1$ for all non-negative integers $n$, we have the $A^{r}$ method; it has been introduced by Başar [6] (see also [1], [2], [3], [4], and [5] for some results related to sequence spaces defined by the domain of the $A^{r}$ matrices). The recent monograph [10] is devoted to the sequence spaces, summability theory and on the domain of certain triangle matrices in the normed/paranormed sequence spaces. In [12], Talo and Başar have given necessary and sufficient Tauberian conditions for the $A^{r}$ method. In this paper, we extend the results of [12] to $A^{r, p}$ and obtain necessary and sufficient conditions for the summability method $A^{r, p}$ under which the existence of the limit (3) follows from that of (2).

2. Auxiliary Results. We need the following lemmas to prove our theorems.

Denote the integer part of the product $\lambda$ and $n$ by $\lambda_{n}:=[\lambda n]$.

Lemma 1. [8], [9] If $\left(p_{n}\right)$ is a sequence of non-negative numbers, the conditions

$$
\limsup _{n \rightarrow \infty} \frac{P_{n}}{P_{\lambda_{n}}}<1 \quad \text { for every } \quad \lambda>1
$$

and

$$
\limsup _{n \rightarrow \infty} \frac{P_{\lambda_{n}}}{P_{n}}<1 \quad \text { for every } \quad 0<\lambda<1
$$


are equivalent.

Lemma 2. Let (4) be satisfied. If a sequence $\left(x_{n}\right)$ is $A^{r, p}$ summable to a finite number $l$, then

$$
\lim _{n \rightarrow \infty} \frac{1}{P_{\lambda_{n}}-P_{n}} \sum_{k=n+1}^{\lambda_{n}} p_{k}\left(1+r^{k}\right) x_{k}=l \quad \text { for every } \quad \lambda>1
$$

and

$$
\lim _{n \rightarrow \infty} \frac{1}{P_{n}-P_{\lambda_{n}}} \sum_{k=\lambda_{n}+1}^{n} p_{k}\left(1+r^{k}\right) x_{k}=l \quad \text { for every } \quad 0<\lambda<1 .
$$

Proof. Case $\lambda>1$. By definition,

$$
\frac{1}{P_{\lambda_{n}}-P_{n}} \sum_{k=n+1}^{\lambda_{n}} p_{k}\left(1+r^{k}\right) x_{k}=\sigma_{n, p}^{r}+\frac{P_{\lambda_{n}}}{P_{\lambda_{n}}-P_{n}}\left(\sigma_{\lambda_{n}, p}^{r}-\sigma_{n, p}^{r}\right) .
$$

By (4), we have

$$
0<\limsup _{n \rightarrow \infty} \frac{P_{\lambda_{n}}}{P_{\lambda_{n}}-P_{n}}=\left(1-\limsup _{n \rightarrow \infty} \frac{P_{n}}{P_{\lambda_{n}}}\right)^{-1}<\infty .
$$

Now, (6) follows from (2) and (8).

Case $0<\lambda<1$. By definition,

$$
\frac{1}{P_{n}-P_{\lambda_{n}}} \sum_{k=\lambda_{n}+1}^{n} p_{k}\left(1+r^{k}\right) x_{k}=\sigma_{n, p}^{r}+\frac{P_{n}}{P_{n}-P_{\lambda_{n}}}\left(\sigma_{\lambda_{n}, p}^{r}-\sigma_{n, p}^{r}\right) .
$$

From (5) we have

$$
0<\limsup _{n \rightarrow \infty} \frac{P_{n}}{P_{n}-P_{\lambda_{n}}}=\left(1-\limsup _{n \rightarrow \infty} \frac{P_{\lambda_{n}}}{P_{n}}\right)^{-1}<\infty .
$$

Now, (7) follows from (2) and (9).

3. Main Results. First, we consider sequences of real numbers and prove the following one-sided Tauberian theorem.

Theorem 1. Let (4) be satisfied, $\left(x_{n}\right)$ be a sequence of real numbers, $A^{r, p}$-summable to a finite limit $l$; then (3) holds if and only if the following two conditions are satisfied:

$$
\sup _{\lambda>1} \liminf _{n \rightarrow \infty} \frac{1}{P_{\lambda_{n}}-P_{n}} \sum_{k=n+1}^{\lambda_{n}}\left(p_{k}\left(1+r^{k}\right) x_{k}-p_{k} x_{n}\right) \geqslant 0
$$


and

$$
\sup _{0<\lambda<1} \liminf _{n \rightarrow \infty} \frac{1}{P_{n}-P_{\lambda_{n}}} \sum_{k=\lambda_{n}+1}^{n}\left(p_{k} x_{n}-p_{k}\left(1+r^{k}\right) x_{k}\right) \geqslant 0 .
$$

A sequence $\left(x_{n}\right)$ of real numbers is said to be slowly decreasing if

$$
\lim _{\lambda \rightarrow 1^{+}} \liminf _{n \rightarrow \infty} \min _{n<k \leqslant \lambda_{n}}\left(x_{k}-x_{n}\right) \geqslant 0 .
$$

Note that condition (12) can be equivalently reformulated as:

$$
\lim _{\lambda \rightarrow 1^{-}} \liminf _{n \rightarrow \infty} \min _{\lambda_{n}<k \leqslant n}\left(x_{n}-x_{k}\right) \geqslant 0 .
$$

The right-hand limit in (12) exists and can be equivalently replaced by $\sup _{\lambda>1}$. The concept of slow decreasing was introduced by Schmidt [11].

For sequences $\left(x_{n}\right)$ and $\left(y_{n}\right)$ of real or complex numbers, we write $x_{n}=O\left(y_{n}\right)$ if there exists some positive number $M$, such that $\left|x_{n}\right| \leqslant M\left|y_{n}\right|$ for all sufficiently large $n$.

We have the following corollary for Theorem 1.

Corollary 1. Let (4) and $P_{n}=O\left(n p_{n}\right)$ be satisfied. If a sequence $\left(x_{n}\right)$ of real numbers is slowly decreasing, (2) implies (3).

Remark. If conditions (2) and (3) or, equivalently, the conditions (2), (10), and (11) are satisfied, then we necessarily have

$$
\lim _{n \rightarrow \infty} \frac{1}{P_{\lambda_{n}}-P_{n}} \sum_{k=n+1}^{\lambda_{n}}\left(p_{k}\left(1+r^{k}\right) x_{k}-p_{k} x_{n}\right)=0
$$

for every $\lambda>1$ and

$$
\lim _{n \rightarrow \infty} \frac{1}{P_{n}-P_{\lambda_{n}}} \sum_{k=\lambda_{n}+1}^{n}\left(p_{k} x_{n}-p_{k}\left(1+r^{k}\right) x_{k}\right)=0
$$

for every $0<\lambda<1$.

Remark. Theorem 1 remains true if conditions (10) and (11) are replaced by their symmetric counterparts:

$$
\inf _{\lambda>1} \limsup _{n \rightarrow \infty} \frac{1}{P_{\lambda_{n}}-P_{n}} \sum_{k=n+1}^{\lambda_{n}}\left(p_{k}\left(1+r^{k}\right) x_{k}-p_{k} x_{n}\right) \leqslant 0
$$


and

$$
\inf _{0<\lambda<1} \limsup _{n \rightarrow \infty} \frac{1}{P_{n}-P_{\lambda_{n}}} \sum_{k=\lambda_{n}+1}^{n}\left(p_{k} x_{n}-p_{k}\left(1+r^{k}\right) x_{k}\right) \leqslant 0,
$$

respectively.

Second, we consider sequences of complex numbers and prove the following two-sided Tauberian theorem.

Theorem 2. Let (4) be satisfied, $\left(x_{n}\right)$ be a $A^{r, p}$-summable sequence of complex numbers; then $\left(x_{n}\right)$ converges to the same limit if and only if one of the following two conditions is satisfied:

$$
\inf _{\lambda>1} \limsup _{n \rightarrow \infty}\left|\frac{1}{P_{\lambda_{n}}-P_{n}} \sum_{k=n+1}^{\lambda_{n}}\left(p_{k}\left(1+r^{k}\right) x_{k}-p_{k} x_{n}\right)\right|=0
$$

or

$$
\inf _{0<\lambda<1} \limsup _{n \rightarrow \infty}\left|\frac{1}{P_{n}-P_{\lambda_{n}}} \sum_{k=\lambda_{n}+1}^{n}\left(p_{k} x_{n}-p_{k}\left(1+r^{k}\right) x_{k}\right)\right|=0 .
$$

A sequence $\left(x_{n}\right)$ of complex numbers is said to be slowly oscillating if

$$
\lim _{\lambda \rightarrow 1^{+}} \limsup _{n \rightarrow \infty} \max _{n<k \leqslant \lambda_{n}}\left|x_{k}-x_{n}\right|=0 .
$$

The concept of slow oscillation was introduced by Hardy [7]. An equivalent reformulation of (20) can be given as follows:

$$
\lim _{\lambda \rightarrow 1^{-}} \limsup _{n \rightarrow \infty} \max _{\lambda_{n}<k \leqslant n}\left|x_{k}-x_{n}\right|=0 .
$$

The right-hand limit in (20) can be equivalently replaced by $\inf _{\lambda>1}$.

We have the following corollary for Theorem 2:

Corollary 1. Let (4) and $P_{n}=O\left(n p_{n}\right)$ be satisfied. If a sequence $\left(x_{n}\right)$ of complex numbers is slowly oscillating, (2) implies (3).

4. Proofs. In this section we present the proofs. Proof of Theorem 1.

Necessity. Assume that (2), (3), and (4) are satisfied. Then Lemma 4 yields (10) in case $\lambda>1$ and (11) in case $0<\lambda<1$.

Sufficiency. Assume that (2), (4), (10) and (11) are satisfied. 
First, consider the case $\lambda>1$. Let $\epsilon>0$ be given. By (10), there exists some $\lambda>1$, such that

$$
\liminf _{n \rightarrow \infty} \frac{1}{P_{\lambda_{n}}-P_{n}} \sum_{k=n+1}^{\lambda_{n}}\left(p_{k}\left(1+r^{k}\right) x_{k}-p_{k} x_{n}\right) \geqslant-\epsilon .
$$

It follows from (8) that

$$
\begin{aligned}
x_{n}-\sigma_{n, p}^{r}=\frac{P_{\lambda_{n}}}{P_{\lambda_{n}}-P_{n}}\left(\sigma_{\lambda n, p}^{r}-\sigma_{n, p}^{r}\right)- & \\
& \quad-\frac{1}{P_{\lambda_{n}}-P_{n}} \sum_{k=n+1}^{\lambda_{n}}\left(p_{k}\left(1+r^{k}\right) x_{k}-p_{k} x_{n}\right) .
\end{aligned}
$$

By (4), we have

$$
\lim _{n \rightarrow \infty} \frac{P_{\lambda_{n}}}{P_{\lambda_{n}}-P_{n}}\left(\sigma_{\lambda_{n}, p}^{r}-\sigma_{n, p}^{r}\right)=0 .
$$

Combining (23) and (24) gives

$$
\begin{aligned}
& \limsup _{n \rightarrow \infty}\left(x_{n}-\sigma_{n, p}^{r}\right) \leqslant \limsup _{n \rightarrow \infty} \frac{P_{\lambda_{n}}}{P_{\lambda_{n}}-P_{n}}\left(\sigma_{\lambda_{n}, p}^{r}-\sigma_{n, p}^{r}\right)+ \\
& +\limsup _{n \rightarrow \infty}\left(-\frac{1}{P_{\lambda_{n}}-P_{n}} \sum_{k=n+1}^{\lambda_{n}}\left(p_{k}\left(1+r^{k}\right) x_{k}-p_{k} x_{n}\right)\right) \leqslant \\
& \leqslant-\liminf _{n \rightarrow \infty}\left(\frac{1}{P_{\lambda_{n}}-P_{n}} \sum_{k=n+1}^{\lambda_{n}}\left(p_{k}\left(1+r^{k}\right) x_{k}-p_{k} x_{n}\right)\right) \leqslant \epsilon .
\end{aligned}
$$

Consequently, we have

$$
\limsup x_{n} \leqslant l+\epsilon \text {. }
$$

Second, consider the case $0<\lambda<1$. It follows from (9) that

$$
\begin{aligned}
x_{n}-\sigma_{n, p}^{r}=\frac{P_{n}}{P_{n}-P_{\lambda_{n}}}( & \left.\sigma_{n, p}^{r}-\sigma_{\lambda_{n}, p}^{r}\right)+ \\
& +\frac{1}{P_{n}-P_{\lambda_{n}}} \sum_{k=\lambda_{n}+1}^{n}\left(p_{k} x_{n}-p_{k}\left(1+r^{k}\right) x_{k}\right) .
\end{aligned}
$$


Using a similar argument as above, we conclude by (5) and (11) for any given $\epsilon>0$ that

$$
\begin{aligned}
\liminf _{n \rightarrow \infty}\left(x_{n}-\right. & \left.\sigma_{n, p}^{r}\right) \geqslant \liminf _{n \rightarrow \infty} \frac{P_{\lambda_{n}}}{P_{n}-P_{\lambda_{n}}}\left(\sigma_{n, p}^{r}-\sigma_{\lambda_{n}, p}^{r}\right)+ \\
& +\liminf _{n \rightarrow \infty}\left(\frac{1}{P_{n}-P_{\lambda_{n}}} \sum_{k=\lambda_{n}+1}^{n}\left(p_{k} x_{n}-p_{k}\left(1+r^{k}\right) x_{k}\right)\right) \geqslant-\epsilon .
\end{aligned}
$$

Consequently, we have

$$
\liminf _{n \rightarrow \infty} x_{n} \geqslant l-\epsilon
$$

Combining (15) and (27) yields

$$
l-\epsilon \leqslant \liminf _{n \rightarrow \infty} x_{n} \leqslant \limsup _{n \rightarrow \infty} x_{n} \leqslant l+\epsilon .
$$

Choose $\epsilon$ arbitrary small; hence (3) follows.

Proof of Corollary 1. For $\lambda>1$, we have the following inequality:

$$
\begin{aligned}
& \frac{1}{P_{\lambda_{n}}-P_{n}} \sum_{k=n+1}^{\lambda_{n}}\left(p_{k}\left(1+r^{k}\right) x_{k}-p_{k} x_{n}\right) \geqslant \min _{n<k \leqslant \lambda_{n}}\left(\left(1+r^{k}\right) x_{k}-x_{n}\right) \geqslant \\
& \geqslant \min _{n<k \leqslant \lambda_{n}}\left(x_{k}-x_{n}\right)+\min _{n<k \leqslant \lambda_{n}}\left(r^{k} x_{k}\right) .
\end{aligned}
$$

We have

$$
x_{n}=\frac{P_{n} \sigma_{n, p}^{r}-P_{n-1} \sigma_{n-1, p}^{r}}{p_{n}\left(1+r^{n}\right)}
$$

and

$$
\frac{x_{n}}{n}=\frac{P_{n}\left(\sigma_{n, p}^{r}-\sigma_{n-1, p}^{r}\right)}{n p_{n}\left(1+r^{n}\right)}+\frac{\sigma_{n-1, p}^{r}}{n\left(1+r^{n}\right)} .
$$

Since $\left(x_{n}\right)$ is summable $A^{r, p}$ and $P_{n}=O\left(n p_{n}\right)$, we have $\frac{x_{n}}{n} \rightarrow 0$ as $n \rightarrow \infty$. Therefore, $r^{n} x^{n} \rightarrow 0$ as $n \rightarrow \infty$. Hence, condition (12) clearly implies (10). Similarly, (13) implies (11). By Theorem 1, we have (3).

Proof of Theorem 2.

Necessity. The proof is similar to the proof of the necessity part of Theorem 1. 
Sufficiency. Assume that (2) and one of the conditions (18) and (19) are satisfied. Let any $\epsilon>0$ be given. By (18), there exists some $\lambda>1$, such that

$$
\limsup _{n \rightarrow \infty}\left|\frac{1}{P_{\lambda_{n}}-P_{n}} \sum_{k=n+1}^{\lambda_{n}}\left(p_{k}\left(1+r^{k}\right) x_{k}-p_{k} x_{n}\right)\right|<\epsilon .
$$

By (23), we have

$$
\begin{aligned}
\limsup _{n \rightarrow \infty}\left|x_{n}-\sigma_{n, p}^{r}\right| \leqslant \limsup _{n \rightarrow \infty} \frac{P_{\lambda_{n}}}{P_{\lambda_{n}}-P_{n}}\left|\sigma_{\lambda_{n}, p}^{r}-\sigma_{n, p}^{r}\right|+ \\
\quad+\limsup _{n \rightarrow \infty}\left|\frac{1}{P_{\lambda_{n}}-P_{n}} \sum_{k=n+1}^{\lambda_{n}}\left(p_{k}\left(1+r^{k}\right) x_{k}-p_{k} x_{n}\right)\right| .
\end{aligned}
$$

By (19), there exists some $0<\lambda<1$, such that

$$
\limsup _{n \rightarrow \infty}\left|\frac{1}{P_{n}-P_{\lambda_{n}}} \sum_{k=\lambda_{n}+1}^{n}\left(p_{k} x_{n}-p_{k}\left(1+r^{k}\right) x_{k}\right)\right|<\epsilon .
$$

By (26), we have

$$
\begin{aligned}
\limsup _{n \rightarrow \infty}\left|x_{n}-\sigma_{n, p}^{r}\right| \leqslant \limsup _{n \rightarrow \infty} \frac{P_{n}}{P_{n}-P_{\lambda_{n}}}\left|\sigma_{n, p}^{r}-\sigma_{\lambda_{n}, p}^{r}\right|+ \\
+\limsup _{n \rightarrow \infty}\left|\frac{1}{P_{n}-P_{\lambda_{n}}} \sum_{k=\lambda_{n}+1}^{n}\left(p_{k} x_{n}-p_{k}\left(1+r^{k}\right) x_{k}\right)\right|
\end{aligned}
$$

By (29) or (31), in either case we obtain

$$
\limsup _{n \rightarrow \infty}\left|x_{n}-\sigma_{n, p}^{r}\right|=0
$$

whence, it follows that

$$
\lim _{n \rightarrow \infty}\left|x_{n}-\sigma_{n, p}^{r}\right|=0 .
$$

Now, we conclude (3) from (2) and (33).

Proof of Corollary 1. For $\lambda>1$, we have the following inequality:

$$
\left|\frac{1}{P_{\lambda_{n}}-P_{n}} \sum_{k=n+1}^{\lambda_{n}}\left(p_{k}\left(1+r^{k}\right) x_{k}-p_{k} x_{n}\right)\right| \leqslant \max _{n<k \leqslant \lambda_{n}}\left|\left(1+r^{k}\right) x_{k}-x_{n}\right| \leqslant
$$




$$
\leqslant \max _{n<k \leqslant \lambda_{n}}\left|x_{k}-x_{n}\right|+\max _{n<k \leqslant \lambda_{n}}\left|r^{k} x_{k}\right|
$$

As in the proof of Corollary 1, we have $\frac{x_{n}}{n} \rightarrow 0$ as $n \rightarrow \infty$. Therefore, $r^{n} x^{n} \rightarrow 0$ as $n \rightarrow \infty$. Hence, condition (20) clearly implies (18). Similarly, (21) implies (19). By Theorem 2, we have (3).

Acknowledgment. The authors would like to thank the anonymous referee for his/her careful reading of the manuscript, correcting many errors, and useful comments that improved the manuscript.

\section{References}

[1] Aydın C., Başar F. On the new sequence spaces which include the spaces $c_{0}$ and $c$. Hokkaido Math. J., 2004, vol. 33, no. 2, pp. 383-398. DOI: https://doi.org/10.14492/hokmj/1285766172

[2] Aydın C., Başar F. Some new paranormed sequence spaces. Inform Sci., 2004, vol. 160, no. 1-4, pp. 27-40. DOI: https://doi.org/10.1016/j.ins.2003.07.009

[3] Aydın C., Başar F. Some new difference sequence spaces. Appl. Math. Comput., 2004, vol. 157, no. 3, pp. 677-693. DOI: https://doi.org/10.1016/j.amc.2003.08.055

[4] Aydın C., Başar F. Some new sequence spaces which include the spaces $\ell_{p}$ and $\ell_{\infty}$. Demonstratio Math., 2005, vol. 38, no. 3, pp. 641-656. DOI: https://doi.org/10.1515/dema-2005-0313

[5] Aydın C., Başar F. Some generalizations of the sequence space $a_{p}^{r}$. Iran. J. Sci. Technol. Trans. A. Sci., 2006, vol. 30, no. A2, 175-190. DOI: https://dx.doi.org/10.22099/ijsts.2006.2746

[6] Başar F. A note on the triangle limitation methods. Firat Univ. Fen. \& Müh. Bil. Dergisi, 1993, vol. 5, no. 1, pp. 113-117.

[7] Hardy G.H. Divergent series. Chalsea, New York, 1991.

[8] Kambak Ç., Çanak I. An alternative proof of a Tauberian theorem for the weighted mean method of summability. Natl. Acad. Sci. Lett., 2019, vol. 42, no. 4, pp. 355-357. DOI: https://doi .org/10.1007/s40009-018-0754-7

[9] Moricz F., Rhoades B.E. Necessary and sufficient Tauberian conditions for certain weighted mean methods of summability. II. Acta Math. Hungar., 2004, vol. 102, no. 4, pp. 279-285. DOI: https://doi.org/10.1007/BF01874356

[10] Mursaleen M., Başar F. Sequence Spaces: Topics in Modern Summability Theory. CRC Press, Taylor \& Francis Group, Series: Mathematics and Its Applications, Boca Raton, London, New York, 2020. 
[11] Schmidt R. Über divergente Folgen und lineare Mittelbildungen. Math. Z., 1925, vol. 22, pp. 89-152. DOI: https://doi.org/10.1007/BF01479600

[12] Talo Ö., Başar F. Necessary and sufficient Tauberian conditions for the $A^{r}$ method of summability. Math. J. Okayama Univ., 2018, vol. 60, pp. 209219 .

Received March 25, 2021.

In revised form, April 23, 2021.

Accepted April 25, 2021.

Published online May 21, 2021.

Ege University

Faculty of Science, Department of Mathematics

Erzene District, Bornova/İzmir 35040, Turkey

E-mail: caglakambak@gmail.com

Ege University

Faculty of Science, Department of Mathematics

Erzene District, Bornova/İzmir 35040, Turkey

E-mail: ibrahim.canak@ege.edu.tr 